\title{
Malpighian Tubule Polytene Chromosomes of Culex quinquefasciatus (Diptera, Culicinae)
}

\author{
Jairo Campos $/{ }^{+}$, Carlos Fernando S Andrade, Shirlei M Recco-Pimentel*
}

\author{
Departamento de Zoologia *Departamento de Biologia Celular, Instituto de Biologia, Universidade Estadual de Campinas, \\ 13084-971 Campinas, SP, Brasil
}

\begin{abstract}
Dipteran polytene chromosomes provide an excellent model for understanding in species complexes, as well as for structural and functional cytogenetics. The status of species in the Culex pipiens complex is controversial and the use of polytene chromosomes for cytogenetic analysis in the subfamily Culicinae has been difficult because of methodological problems. In this study, Malpighian tubule polytene chromosomes were obtained from young (0 to $\left.12 \mathrm{~h}, 20^{\circ} \mathrm{C}\right)$ and old $\left(20\right.$ to $\left.42 \mathrm{~h}, 28^{\circ} \mathrm{C}\right)$ laboratory-bred C. pipiens quinquefasciatus pupae. The chromosome maps for this species were constructed and compared with published data for C. pipiens pipiens and C. p. quinquefasciatus. Although the banding patterns were conserved between subspecies, analysis of the structural variations in the bands and interbands revealed differences apparently related to the physiological stage and ecogeographical strain. The organization of the centromeric regions in larval and pupal chromosomes showed greater similarity to each other than did those of pupal and adult chromosomes. The use of pupal polytene chromosomes for in situ hybridization with vector competence probes is discussed.
\end{abstract}

Key words: cytogenetic - Culex pipiens - insecticide resistance - mosquitoes

The cytogenetics and molecular biology of the Culicidae can be studied by analyzing their polytene chromosome structure. Culex pipiens is a species complex (Sirivanakarn \& White 1978, Hemingway \& Karunaratne 1998) that includes C. pipiens Linnaeus, 1758 (s.str.), C.p. quinquefasciatus Say, 1823 and C. p. molestus Forskal, 1775 , which differ from each other primarily in their physiology, behavior, and distribution (Olejnícek \& Gelbic 2000). Salivary gland polytene chromosomes have been used in cytogenetic analyses of this complex (Sutton 1942, Kitzmiller \& Clark 1952, Kitzmiller \& Keppler 1961, Dennhöfer 1968, Kanda 1970, Tewfik \& Barr 1974, Verma et al. 1987). Although such analyses are of limited use for some species of mosquitoes (e.g. Aedes aegypti), Malpighian tubule polytene chromosomes are excellent material for the detailed cytogenetic analysis of $C$. quinquefasciatus (Achary 1994, Campos 2002) and C. pipiens (Zambetaki et al. 1998). In molecular cytogenetic studies of Culex, salivary gland chromosomes have been used to locate the genes related to insecticide resistance (Heyse et al. 1996, Tomita et al. 1996). In the present study, pupal Malpighian tubule polytene chromosome maps were obtained for C. quinquefasciatus and compared with published data for other stages and organs of $C$. pipiens and C. quinquefasciatus.

This research was supported by Capes.

${ }^{+}$Corresponding author. Fax: +55-19-3289.3124. E-mail: jairocag@yahoo.com,cfeandra@unicamp.br

Received 26 August 2002

Accepted 31 January 2003

\section{MATERIALS AND METHODS}

The mosquitoes were collected in July 1999 and February 2001, and the colonies (Unicamp resistant strains UNI-R1 and UNI-R2) were maintained in the Department of Zoology at the State University of Campinas. Polytene chromosome preparations were obtained using larval, pupal and adult female Malpighian tubules. The insects were reared under standard conditions $\left(28 \pm 1{ }^{\circ} \mathrm{C}, 70 \pm\right.$ $10 \%$ relative humidity) or at different temperatures (20$25^{\circ} \mathrm{C}$ ). Following dissection in Ringer's solution, the Malpighian tubules were transferred to a siliconized coverglass with aceto-alcohol (1:3) for 60-70 sec and subsequently stained with $2 \%$ lacto-aceto-orcein for 2-5 $\mathrm{min}$. The cells were dissected in lactoacetic acid (85\% lactic acid- $100 \%$ acetic acid, $55: 45, \mathrm{v} / \mathrm{v}$ ) then $60 \%$ acetic acid was added. This process was completed in about $10 \pm 2$ min. Squashing was done by gently tapping the covership surface. The pupal chromosome maps were constructed based on published photographs and line drawings of $C$. pipiens and C. quinquefasciatus polytene chromosomes. The pupal maps were compared with larval (salivary gland) and adult (Malpighian tubule) maps or photographs of $C$. pipiens (Dennhöfer 1968, 1974, Tewfik \& Barr 1974, Heyse et al. 1996, Zambetaki et al. 1998) and C. quinquefasciatus (Kanda 1970, Verma et al. 1987, Achary 1994, Heyse et al. 1996). The IAL-standard susceptible strain (Adolfo Lutz Institute, IAL-S) originating from Iguape, SP, 1983 (Bracco et al. 1999) was used as a control.

\section{RESULTS}

The Malpighian tubules of pupae $\left(2 \pm 2 \mathrm{~h}, 20^{\circ} \mathrm{C} ; 24 \pm\right.$ 2 and $30 \pm 2 \mathrm{~h}$ old, $28^{\circ} \mathrm{C}$ ) provided the best cytogenetic analysis. The photographic maps of the pupal Malpighian tubule polytene chromosomes are shown in Figs 1 and 2. The polytene chromosomes were numbered 1, 2 and 3 for the shortest, the intermediate and the longest chromosomes respectively. The polytene chromosome comple- 
ment was divided into 70 sections based on other polytene maps of the $C$. pipiens complex (Kanda 1970, Tewfik \& Barr 1974, Verma et al. 1987, Zambetaki et al. 1998). The maps were labeled according to Dennhöfer $(1968,1974)$, with 16, 25 and 29 sections for chromosomes 1, 2 and 3, respectively.

The Malpighian chromosome complement consisted of three pairs of synapsed, banded chromosomes, although asynaptic regions have also been reported $(3,4-$ 1R; 26-2R, 30-2L; 62/63-3L). Conspicuous centromeric regions, amorphous regions, and puffs characterized the pupal Malpighian tubule polytene chromosomes of $C$. quinquefasciatus. The nuclear organizer region was located in section 10C. Other huge puffs were located in sections 11A, 12D, 36A, 53C, 55D and 61D.

Fig. 3 shows the third polytene chromosome of adult Malpighian tubules (21-day-old female). The arrows in Figs 2 and 3 indicate areas with different levels of expression. Fig. 4 shows pupal chromosome 31-2L sections with amplified (UNI-R1 strain) and unamplified (IAL-S strain) areas.

\section{DISCUSSION}

The banding pattern of polytene chromosomes in the pupal chromosomal maps of $C$. quinquefasciatus showed close similarities to those of maps, photographs and/or drawings of $C$. pipiens and $C$. quinquefasciatus obtained from larval and adult chromosomes. In the present study, were compared two subspecies, three stages (larval, pupal and adult) and two organs (the salivary glands and the Malpighian tubules). The structural differences apparently reflected characteristic physiological processes related to two organs and three stages rather than to huge differences among the subspecies (i.e. C. pipiens and $C$. quinquefasciatus).

The banding patterns of larval (salivary glands) and pupal (Malpighian tubules) chromosomes were more similar to each other than between pupal and adult chromosomes (Malpighian tubules). More similarities were seen in centromeric regions 2 and 3 of pupal and adult $C$. quinquefasciatus than between pupal $C$. quinquefasciatus and adult $C$. pipiens. The similarity of the three centromeric regions in larval and pupal chromosomes was greater than that for pupal and adult chromosomes, independent of the subspecies and strain.

There were marked differences between the band patterns in pupae and those in larval and adult maps, especially in the $2 \mathrm{~L}$ (sections 34 to 36), 3R (section 53) and 3L (section 55/56) arms. The band pattern of sections 57-58 (3L arm) was different in adult $C$. pipiens maps. Variations in band patterns could also result from ecogeographical isolation of the strains. The $31-2 \mathrm{~L}$ section showed an
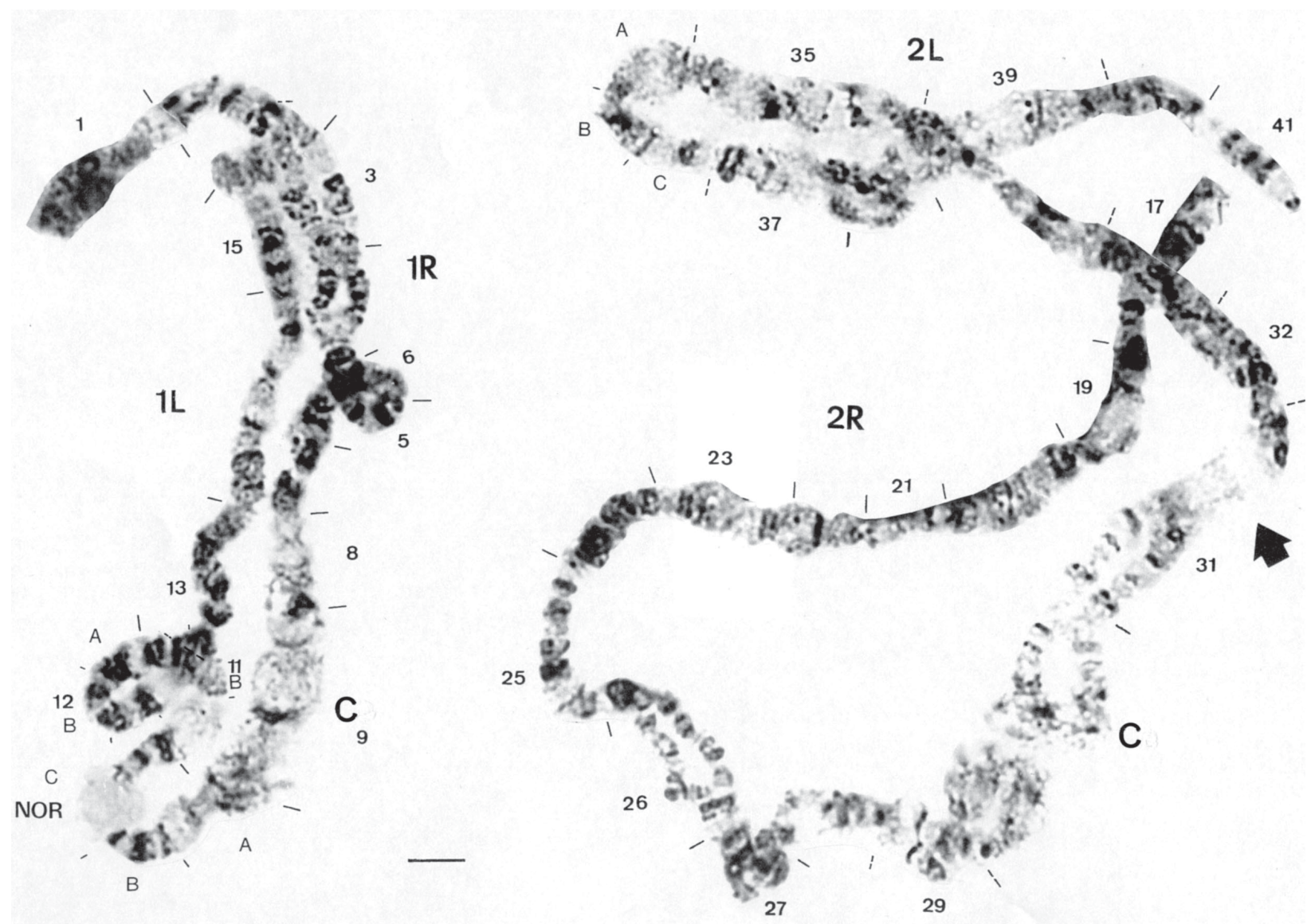

Fig. 1: pupal chromosome maps for polytene chromosomes 1 and 2. L: left arm; R: right arm; C: centromere; NOR: nucleolar organizing region. Arrow: section 31 showing an amorphous area. This region is associated with organophosphorus insecticide resistance genes. Bar $=3.9 \mu \mathrm{m}$ 
amorphous area or a single huge puff in the UNI-R1 (Fig. 4a) and UNI-2 strains. In the IAL-S strain this area is a single diffuse band (Fig. 4b). This amorphous area was also observed in field strains (Cuiabá, MT, and Campo Grande, MS, Brazil, and Palmira, VA, Colombia) (data not shown). This is the region where the esterase genes associated with organophosphorus insecticide resistance are located in C. quinquefasciatus (Heyse et al. 1996) and
C. pipiens (Zambetaki et al. 1998). Tomita et al. (1996) used a TEM-R1 resistant strain primer of $C$. quinquefasciatus in C. pipiens molestus to locate this region on the same $2 \mathrm{~L}$ arm.

Physical maps of polytene chromosome designed to correlate with genetic linkage maps could be developed using fluorescence in situ hybridization (FISH) techniques. This is being done for the metaphase chromosomes of $A$.

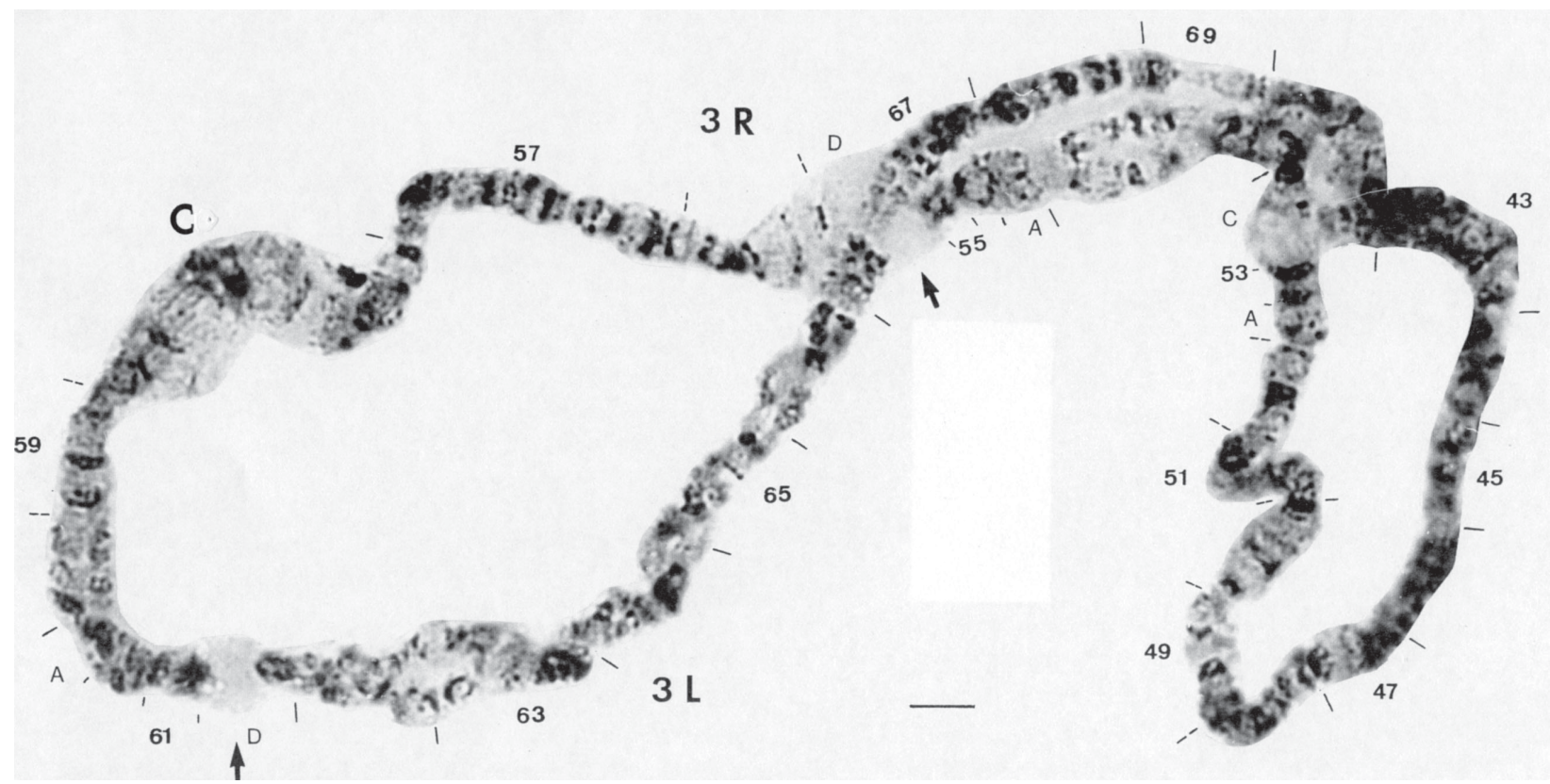

Fig. 2: pupal chromosome map for the third polytene chromosome. L: left arm; R: right arm; C: centromere. The arrows indicate areas with different levels of expression. Bar $=3.9 \mu \mathrm{m}$

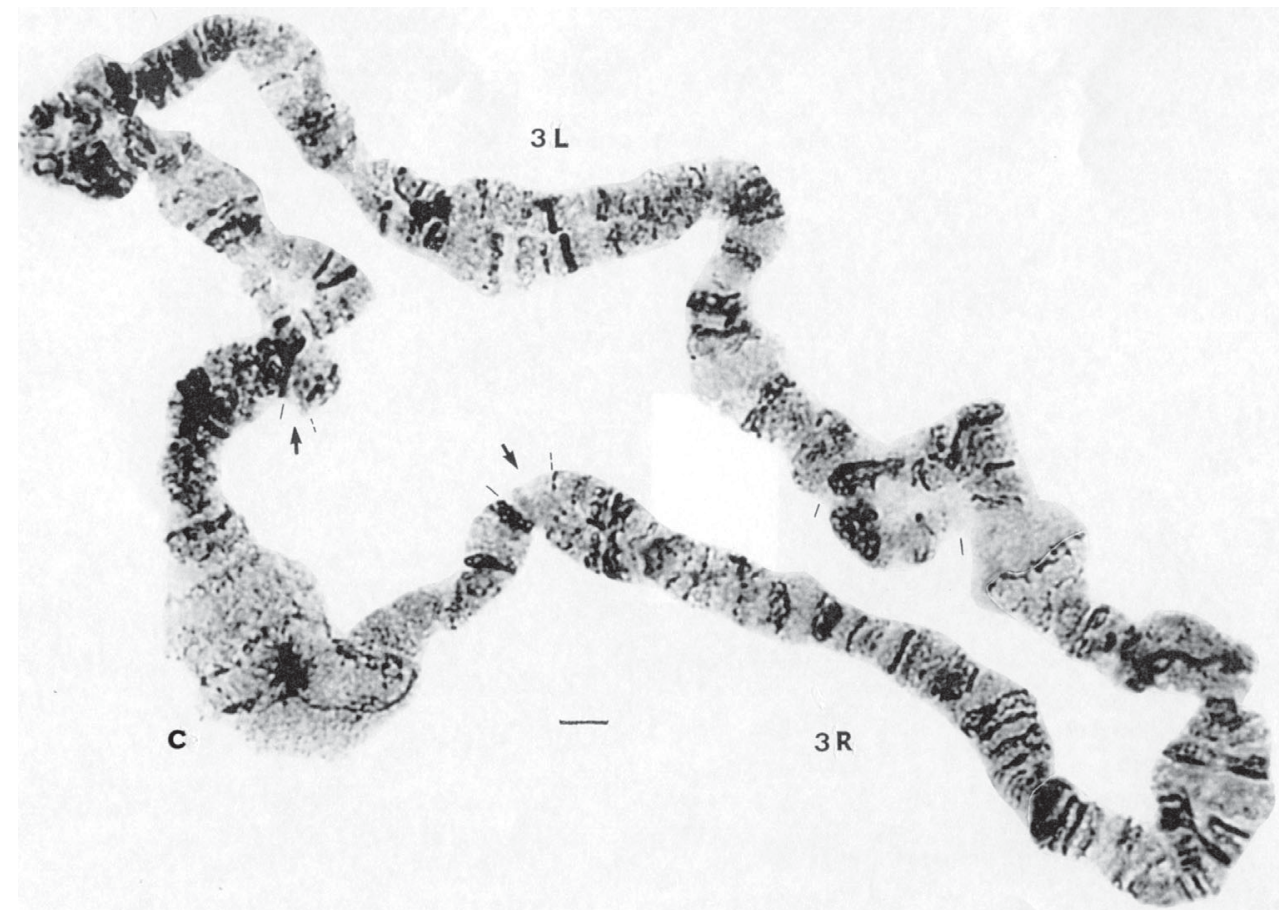

Fig. 3: the third polytene chromosome of adult Malpighian tubules (21-day-old female). L: left arm; R: right arm; C: centromere. The arrows indicate areas with different levels of expression. Bar $=3.9 \mu \mathrm{m}$ 


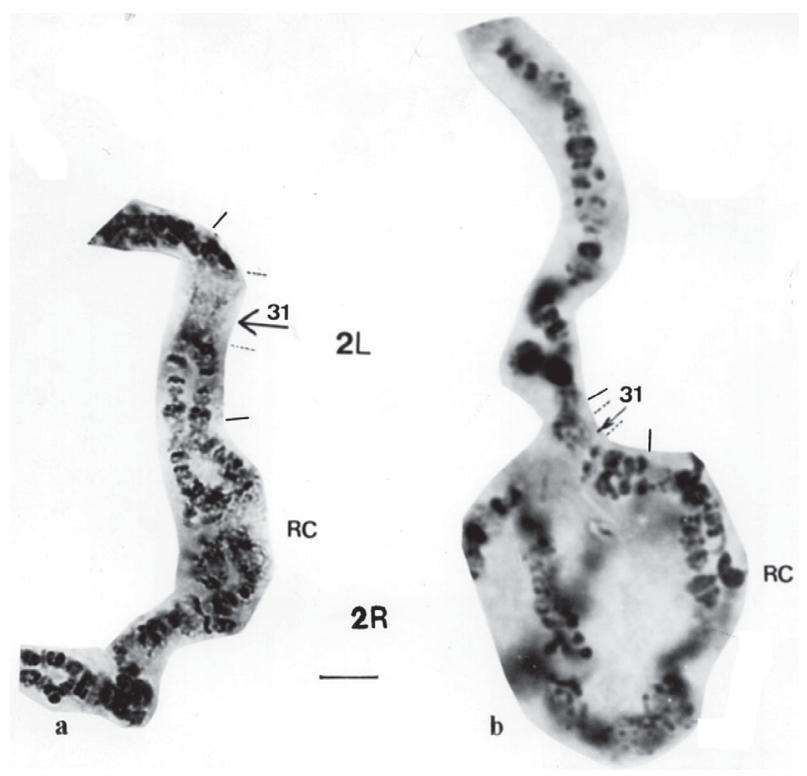

Fig. 4: pupal chromosome 31-2L section of strains UNI-R1 (a) and IAL-S (b). L: left arm; R: right arm; RC: centromere region. The arrows indicate amorphous area and diffuse band, respectively. Bar $=6 \mu \mathrm{m}$ (a) and $4 \mu \mathrm{m}(\mathrm{b})$.

aegypti (Brown \& Knudson 1997, Brown et al. 1995, 2001). The thoracic musculature of mosquitoes is the developmental site for the filarial worms Brugia malayi and $B$. pahangi, and the Malpighian tubules are the developmental site for Dirofilaria immitis. Despite the importance of the thoracic musculature and Malpighian tubules as developmental sites, efforts to identify the gene(s) or gene product(s) responsible for susceptibility or refractoriness to filarial worm infection at these sites have been few (Beerntsen et al. 2000). C. quinquefasciatus is easily reared under laboratory conditions and the procedures for obtainig polytene chromosomes from the larval, pupal and adult stages are well described. Thus, cytogenetic analysis together with the approaches already used for insecticide resistance probes can be applied to research on vector competence and to identify the location of cytogenetic markers.

Larval, pupal and adult Malpighian tubule polytene chromosomes contained structural characteristics suitable for locating resistance and vector competence genes. Chromosome maps are fundamental tools for good cytogenetic analyses of this medically and economically important mosquito. The present description of the polytene chromosomes of $C$. quinquefasciatus provides a cytogenetic basis for future populational studies.

\section{ACKNOWLEDGMENTS}

To Dr Delsio Natal of the Faculty of Public Health, University of São Paulo for providing the Adolfo Lutz Institute standard strain.

\section{REFERENCES}

Achary PMR 1994. A simple technique for the preparation of polytene chromosomes from Culex quinquefasciatus. $\mathrm{J} \mathrm{Am}$ Mosq Control Ass 10: 112-114.

Beerntsen BT, James AA, Christensen BM 2000. Genetics of the mosquito vector competence. Microbiol Mol Biol Rev 64: 115-137.

Bracco JE, Barata JMS, Marinotti O 1999. Evaluation of insecticide resistance in a population of Culex quinquefasciatus (Diptera: Culicidae) from São Paulo, Brazil. Mem Inst Oswaldo Cruz 94: 115-120.

Brown SE, Knudson DL 1997. FISH landmarks for Aedes aegypti chromosomes. Insect Mol Biol 6: 197-202.

Brown SE, Menninger J, Difillipantonio M, Beaty BJ, Ward DC, Knudson DL 1995. Toward a physical map of Aedes aegypti. Insect Mol Biol 4: 161-167.

Brown SE, Severson DW, Smith LA, Knudson DL 2001. Integration of the Aedes aegypti mosquito genetic linkage and physical maps. Genetics 157: 1299-1305.

Campos J 2002. Análise Citológica de Populações de Aedes aegypti (Linnaeus, 1762) e Culex quinquefasciatus Say, 1823 (Diptera, Culicinae), PhD Thesis, Universidade Estadual de Campinas, Campinas, 100 pp.

Dennhöfer L 1968. Die speicheldrüsenchromosomen der stechmücke Culex pipiens I. Der normale chromosomenbestand. Chromosoma 25: 365-376.

Dennhöfer L 1974. Die speicheldrüsenchromosomen der stechmücke Culex pipiens L. II. Ergänzungen zur kartierung. Genetica 45: 29-38.

Hemingway J, Karunaratne SHPP 1998. Mosquito carboxylesterases: a review of the molecular biology and biochemistry of the major insecticide resistance mechanism. Med Vet Entomol 12: 1-12.

Heyse D, Catalan J, Nance E, Britton-Davidian J, Pasteur N 1996. Unconventional organization of amplified esterase B gene in insecticide-resistant mosquitoes of the Culex pipiens complex. J Am Mosq Control Ass 12: 199-205.

Kanda T 1970 . The salivary gland chromosomes of Culex pipiens fatigans Wiedemann. Jpn J Exp Med 40: 335-345.

Kitzmiller JB, Clark CC 1952. Salivary gland chromosomes in Culex mosquitoes. Genetics 37: 596.

Kitzmiller JB, Keppler WJ 1961. Salivary gland chromosomes maps in Culex p. pipiens. Genetics 46: 875-876.

Olejnícek J, Gelbic I 2000. Autogeny in Culex pipiens quinquefasciatus Say. J Vect Ecol 25: 118-122.

Sirivanakarn S, White GB 1978. Neotype designation of Culex quinquefasciatus Say (Diptera: Culicidae). Proc Entomol Soc Wash 80: 360-372.

Sutton E 1942. Salivary gland type chromosomes in mosquitoes. Proc Natl Acad Sci USA 28: 268-272.

Tewfik HR, Barr AR 1974. The salivary gland chromosomes of Culex pipiens L. Mosq News 34: 47-54.

Tomita T, Kono Y, Shimada T 1996. Chromosomal localization of amplified esterase genes in insecticide resistant Culex quinquefasciatus mosquitoes. Insect Biochem Mol Biol 26: 853-857.

Verma RK, Paknaik S, Prasad R, Das CC 1987. Salivary gland chromosomes of Culex quinquefasciatus. Caryologia 40: 99-108.

Zambetaki A, Pasteur N, Mavragani-Tsipidou P 1998. Cytogenetic analysis of the Malpighian tubule polytene chromosomes of Culex pipiens (Diptera: Culicidae). Genome 41: 751-755. 